\title{
Superballistic flow of viscous electron fluid through graphene constrictions
}

\author{
R. Krishna Kumar ${ }^{1,2,3}$, D. A. Bandurin ${ }^{1,2}$, F. M. D. Pellegrino ${ }^{4}$, Y. Cao ${ }^{2}$, A. Principi ${ }^{5}$, H. Guo ${ }^{6}$, \\ G. H. Auton ${ }^{2}$, M. Ben Shalom ${ }^{1,2}$, L. A. Ponomarenko ${ }^{3}$, G. Falkovich ${ }^{7,8}$, K. Watanabe ${ }^{9}$, T. Taniguchi ${ }^{9}$, \\ I. V. Grigorieva', L. S. Levitov ${ }^{6}$, M. Polini ${ }^{1,10}$ and A. K. Geim ${ }^{1,2 \star}$
}

Electron-electron (e-e) collisions can impact transport in a variety of surprising and sometimes counterintuitive ways ${ }^{1-6}$. Despite strong interest, experiments on the subject proved challenging because of the simultaneous presence of different scattering mechanisms that suppress or obscure consequences of e-e scattering ${ }^{7-11}$. Only recently, sufficiently clean electron systems with transport dominated by e-e collisions have become available, showing behaviour characteristic of highly viscous fluids ${ }^{12-14}$. Here we study electron transport through graphene constrictions and show that their conductance below $150 \mathrm{~K}$ increases with increasing temperature, in stark contrast to the metallic character of doped graphene ${ }^{15}$. Notably, the measured conductance exceeds the maximum conductance possible for free electrons ${ }^{16,17}$. This anomalous behaviour is attributed to collective movement of interacting electrons, which 'shields' individual carriers from momentum loss at sample boundaries $^{18,19}$. The measurements allow us to identify the conductance contribution arising due to electron viscosity and determine its temperature dependence. Besides fundamental interest, our work shows that viscous effects can facilitate high-mobility transport at elevated temperatures, a potentially useful behaviour for designing graphene-based devices.

Graphene hosts a high-quality electron system with weak phonon coupling $^{20,21}$ such that $\mathrm{e}-\mathrm{e}$ collisions can become the dominant scattering process at elevated temperatures, $T$. In addition, the electronic structure of graphene inhibits Umklapp processes ${ }^{15}$, which ensures that $\mathrm{e}-\mathrm{e}$ scattering is momentum conserving. These features lead to a fluid-like behaviour of charge carriers, with the momentum taking on the role of a collective variable that governs local equilibrium. Previous studies of the electron hydrodynamics in graphene were carried out using the vicinity geometry and Hall bar devices of a uniform width. Anomalous (negative) voltages were observed, indicating a highly viscous flow, more viscous than that of honey ${ }^{12,22,23}$. In this report, we employ a narrow constriction geometry (Fig. 1a) which offers unique insight into the behaviour of viscous electron fluids. In particular, the hydrodynamic conductance through such constrictions becomes 'superballistic', exceeding the fundamental upper bound allowed in the ballistic limit, which is given by the Sharvin formula ${ }^{16,17}$. This is in agreement with theoretical predictions ${ }^{18,19}$ and is attributed to a peculiar behaviour of viscous flows that self-organize into streams with different velocities, with 'sheaths' of a slow-moving fluid near the constriction edges (Fig. 1b). The cooperative behaviour helps charge carriers to circumnavigate the edges, the only place where the electron flow can lose its net momentum. The physics behind is fairly similar to that involved in the transition from the Knudsen to Poiseuille regimes, well understood in gas dynamics, where the hydrodynamic pressure can rapidly drop upon increasing the gas density and the rate of collisions between molecules ${ }^{24}$.

Our devices are made of monolayer graphene encapsulated between hexagonal boron-nitride crystals, as described in Supplementary Section 1. The device design resembles a multiterminal Hall bar, endowed with constrictions positioned between adjacent voltage probes (Fig. 1c). Below we refer to them as (classical) point contacts (PCs). Five such Hall bars were investigated, each having PCs of various widths $w$ and a reference region without a constriction. The latter allowed standard characterization of graphene, including measurements of its longitudinal resistivity $\rho_{x x}$. All our devices exhibited mobilities exceeding $10 \mathrm{~m}^{2} \mathrm{~V}^{-1} \mathrm{~s}^{-1}$ at liquid-helium $T$, which translates into a mean free path exceeding $1 \mu \mathrm{m}$ with respect to momentum-nonconserving collisions (Supplementary Section 2).

Examples of the measured PC conductance $G_{\mathrm{pc}}$ at $2 \mathrm{~K}$ are given in Fig. 1d. In the low- $T$ regime, all scattering lengths exceed $w$ and transport is ballistic, which allows $G_{p c}$ to be described by the Sharvin formula ${ }^{16}$

$$
G_{\mathrm{b}}=\frac{4 \mathrm{e}^{2}}{h} w \frac{\sqrt{\pi|n|}}{\pi}
$$

where $n$ is the carrier concentration (positive and negative $n$ denote electron and hole doping, respectively). The expression is derived by summing the contributions of individual electron modes that propagate through the constriction, with each of them contributing the conductance quantum, $\mathrm{e}^{2} / h$, towards the total conductance. The dashed curves in Fig. 1d show the PC conductance calculated using equation (1) and assuming the width values, $w_{\mathrm{AFM}}$, as determined by atomic force microscopy (AFM). The observed agreement between the experiment and equation (1) does not rely on any fitting parameters. Alternatively, we could fit our experimental curves using equation (1) and extract the effective width $w$ for each PC (Supplementary Section 3). The results are plotted in the inset of

\footnotetext{
${ }^{1}$ School of Physics and Astronomy, University of Manchester, Manchester M13 9PL, UK. ${ }^{2}$ National Graphene Institute, University of Manchester, Manchester M13 9PL, UK. ${ }^{3}$ Department of Physics, University of Lancaster, Lancaster LA1 4YW, UK. ${ }^{4}$ NEST, Istituto Nanoscienze-CNR and Scuola Normale Superiore, 56126 Pisa, Italy. ${ }^{5}$ Radboud University, Institute for Molecules and Materials, 6525 AJ Nijmegen, the Netherlands. ${ }^{6}$ Massachusetts Institute of Technology, Cambridge, Massachusetts 02139, USA. ${ }^{7}$ Department of Physics, Weizmann Institute of Science, Rehovot 76100 , Israel. ${ }^{8}$ Institute for Information Transmission Problems, Moscow 127994, Russia. ${ }^{9}$ National Institute for Materials Science, 1-1 Namiki, Tsukuba 305-0044, Japan. ${ }^{10}$ Istituto Italiano di Tecnologia, Graphene Labs, Via Morego 30, 16163 Genova, Italy. ${ }^{\star}$ e-mail: geim@manchester.ac.uk
} 

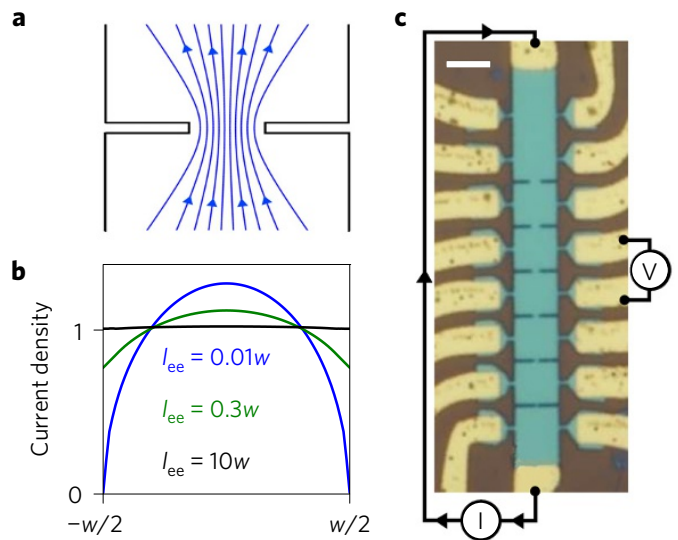
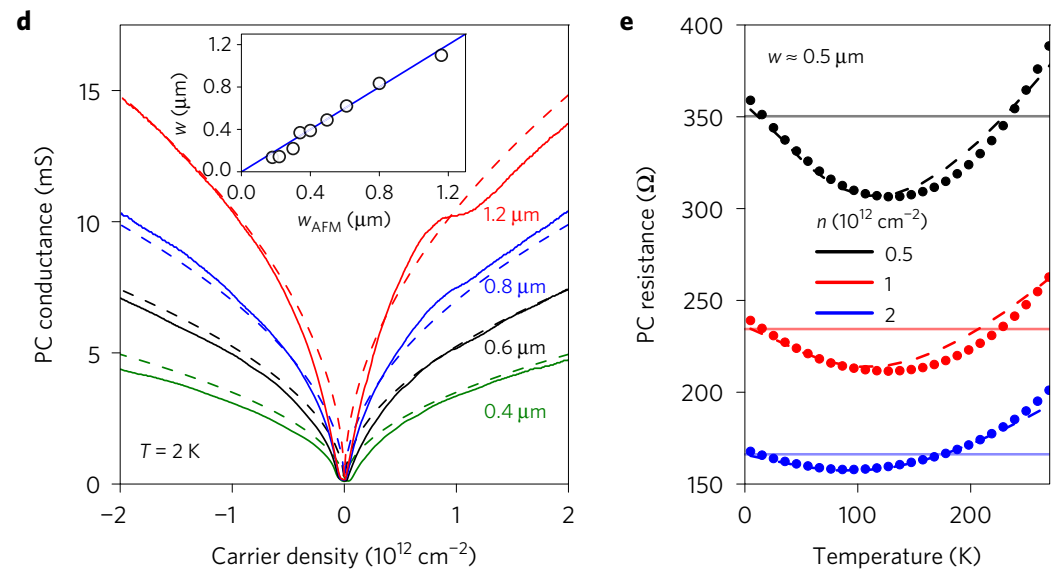

Figure 1 | Electron flow through graphene constrictions. a, Schematic of viscous flow in a PC. $\mathbf{b}$, Distribution of the electric current across the PC, normalized by the total current. In the hydrodynamic regime (e-e scattering length $l_{\text {ee }} \ll w$ ), there is little flow near the edges (blue curve), which reduces the loss of net momentum. Black curve: ballistic regime $l_{\mathrm{ee}} \gg w$. c, Optical micrograph of one of our devices and schematic of our four-probe measurements. Scale bar, $5 \mu \mathrm{m}$. The PCs vary in width from 0.1 to $1.2 \mu \mathrm{m}$. d, Measurements of the low- $T$ conductance for PCs of different $w$ (solid curves). Dashed curves: ballistic conductance given by equation (1). Inset: PC width $w$ found as the best fit to experimental $G_{p c}(n)$ is plotted as a function of $w_{A F M}$.

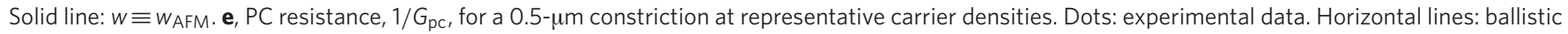
resistance given by equation (1). Dashed curves: theoretical predictions for a viscous electron fluid, using simplified expressions for the $T$ dependence of e-e and electron-phonon scattering ( $\propto T^{2}$ and $T$, respectively). Details are given in Supplementary Section 4.

Fig. $1 \mathrm{~d}$ as a function of $w_{\mathrm{AFM}}$. For $w \geq 0.4 \mu \mathrm{m}$, the agreement between $w$ and $w_{\mathrm{AFM}}$ is within $\sim 5 \%$. Deviations become larger for our smallest constrictions, suggesting that they are effectively narrower, possibly because of edge defects. Although we focus here on classical PCs with a large number of transmitting modes, we note that our devices with $w<0.2 \mu \mathrm{m}$ exhibit certain signs of conductance quantization, fairly similar to those reported previously ${ }^{25,26}$, but they are rapidly washed out upon raising $T$ above $30 \mathrm{~K}$.

The central result of our study is presented in Fig. 1e. It shows that the resistance of graphene PCs, $R_{\mathrm{pc}} \equiv 1 / G_{\mathrm{pc}}$, is a non-monotonic function of $T$, first decreasing as temperature increases. Such $T$ dependence, typical for insulators, is unexpected for our metallic system. It is also in contrast to the $T$ dependence of $\rho_{x x}$ observed in our Hall bar devices. They exhibit $\rho_{x x}$ monotonically increasing with $T$, the standard behaviour in doped graphene (Supplementary Section 2). All our PCs with $w<1 \mu \mathrm{m}$ exhibited this anomalous, insulating-like $T$ dependence up to $100-150 \mathrm{~K}$ (Fig. 2a). As a consequence, $R_{\mathrm{pc}}$ in its minimum corresponds to conductance values that could exceed the ballistic limit by $>15 \%$ (Fig. 1e). At higher $T, R_{\mathrm{pc}}$ starts growing monotonically and follows the same trend as $\rho_{x x}$. The minima in $R_{\mathrm{pc}}(T)$ were more pronounced for narrower constrictions (Fig. 2a), corroborating the importance of the geometry. Figure 2b,c elaborates on the non-metallic behaviour of graphene PCs by plotting maps of the derivative $\mathrm{d} R_{\mathrm{pc}} / \mathrm{d} T$ as a function of both $n$ and $T$. The anomalous insulating-like $T$ dependence shows up as the blue regions whereas the metallic behaviour appears in red. For narrow constrictions, the anomalous behaviour was observed for all accessible $n$ below $100 \mathrm{~K}$, becoming most pronounced at low densities but away from the neutrality point (Figs 1e and 2b). For wide PCs (Fig. 2c), the non-metallic region becomes tiny, in agreement with the expected crossover from the PC to standard Hall bar geometry.

To describe the non-metallic behaviour in our PCs, we first invoke the recent theory ${ }^{18}$ that predicts that e-e scattering modifies equation (1) as

$$
G=G_{\mathrm{b}}+G_{v} \quad \text { where } G_{v}=\frac{\sqrt{\pi|n|} \mathrm{e}^{2} w^{2} v_{\mathrm{F}}}{32 \hbar v}
$$

$v_{\mathrm{F}}$ is the Fermi velocity and e-e collisions are parameterized through the kinematic viscosity $v=v_{\mathrm{F}} l_{\mathrm{ee}} / 4$. The quantity $G_{v}$ is calculated for the Stokes flow through a PC in the extreme hydrodynamic regime (that is, for the e-e scattering length $l_{\mathrm{ee}} \ll w$ ). The additive form of equation (2) is valid ${ }^{18,19}$ for all values of $l_{\mathrm{ee}} / w$, even close to the ballistic regime $l_{\mathrm{ee}} \gg w$. This implies that $G$ should increase with $T$ (in the first approximation ${ }^{15,27}$, as $\propto 1 / l_{\mathrm{ee}} \propto T^{2}$ ), which leads to the initial drop in resistance (Fig. 1e). Equation (2) also suggests that the viscous effects should be more pronounced at low $n$, where electron viscosity is smaller, in agreement with the experiment (Figs $1 \mathrm{e}$ and $2 \mathrm{~b}$ ). The description by equation (2) is valid until phonon scattering becomes significant at higher $T$. To describe both low- $T$ and high- $T$ regimes on an equal footing, we extended the transport model of ref. 18 to account for acousticphonon scattering using an additional term $\propto T$ in the kinetic equation (Supplementary Section 4). The results are plotted in Fig. 1e, showing good agreement with the experiment.

For further analysis, we used our experimental data to extract $G_{v}$, which in turn enabled us to determine $v$ and $l_{\mathrm{ee}}$. To this end, we first followed the standard approach in analysis of transport data for quantum PCs, which takes into account the contact resistance $R_{\mathrm{C}}$ arising from the wide regions leading to constrictions ${ }^{17,28}$. Accordingly, the total resistance of PCs can be represented as

$$
R_{\mathrm{pc}}=\left(G_{\mathrm{b}}+G_{v}\right)^{-1}+R_{\mathrm{C}}
$$

To avoid fitting parameters, we model the contact resistance as $R_{\mathrm{C}}=b \rho_{x x}$, where $b$ is a numerical coefficient calculated by solving the Poisson equation for each specific PC geometry and $\rho_{x x}$ is taken as measured from the reference regions. For our devices, $b$ ranged between 2 and 5 (Supplementary Section 5). Examples of the resulting $\Delta R=R_{\mathrm{pc}}-b \rho_{x x}=\left(G_{\mathrm{b}}+G_{\nu}\right)^{-1}$ are plotted in Fig. 3a. The figure shows that, after the phonon contribution is accounted for through $R_{\mathrm{C}}$, the resistance $\Delta R$ (attributable to the narrowing itself) monotonically decreases with increasing $T$ over the entire $T$ range, in agreement with equation (2). The validity of this analysis is further confirmed by the fact that the extracted values of $\Delta R$ were found to be independent of $b$ (that is, insensitive to voltage probes positions relative to PCs; see Supplementary Fig. 1). As a next step, we use the conductance $G_{\mathrm{b}}$ found in the limit of low $T$ for each PC and subtract this value from $1 / \Delta R=G_{\mathrm{b}}+G_{v}$ to obtain the viscous conductance $G_{v}$. The results are shown in Fig. $3 \mathrm{~b}$ for several PCs. Remarkably, if $G_{v}$ is normalized by $w^{2}$, all the 

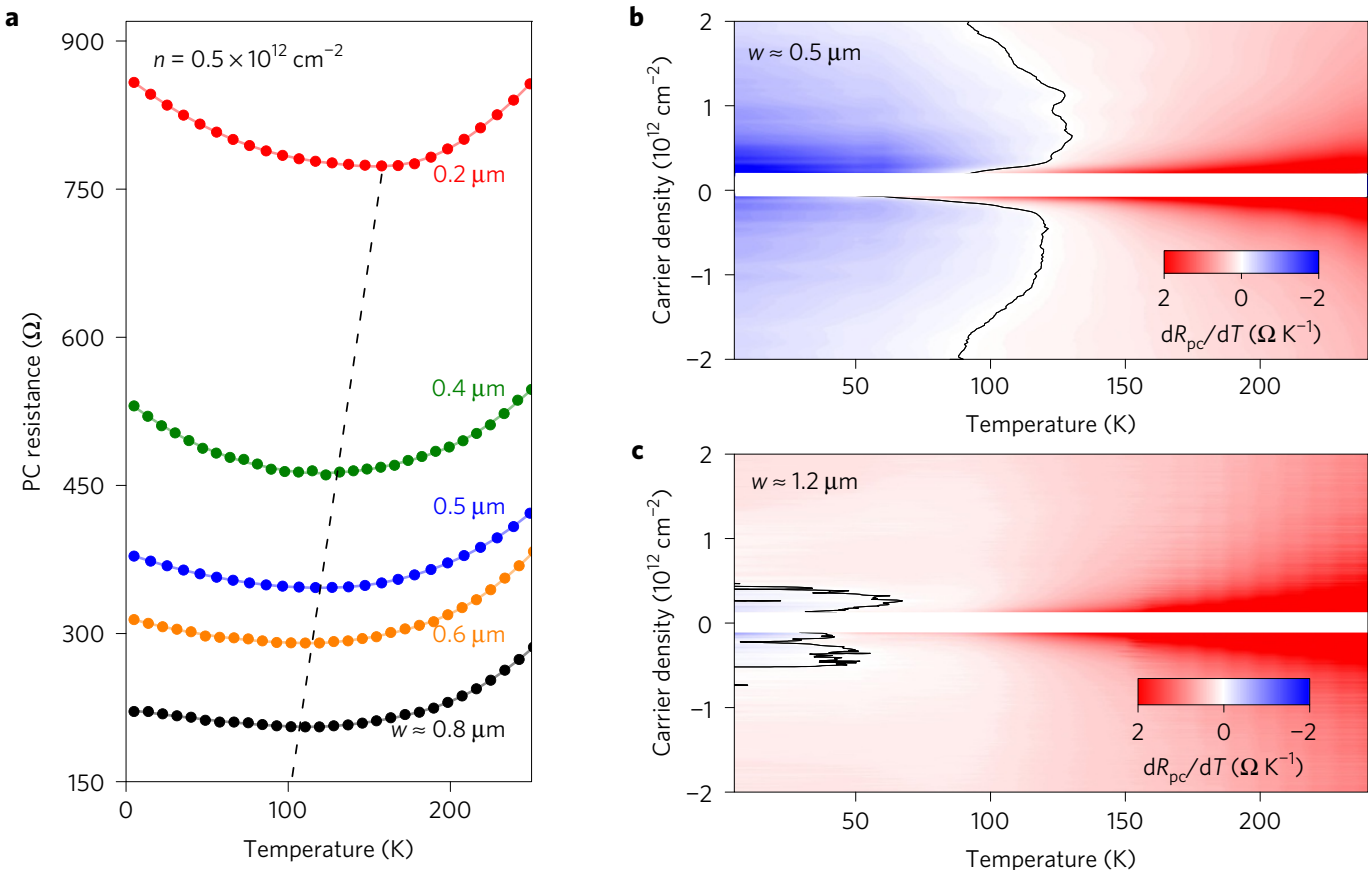

Figure 2 | Transition from metallic to insulating behaviour in constrictions of different widths. a, Temperature dependence for PCs with different $w$ at a given $n$. The dashed line indicates that the minima shift to higher $T$ and become deeper for narrower constrictions. $\mathbf{b}, \mathbf{c}$, Colour map $\mathrm{d} R_{\mathrm{pc}} / \mathrm{d} T(T, n)$ for $w \approx 0.5$ and $1.2 \mu \mathrm{m}$. The black contours mark a transition from the insulating to metallic $T$ dependence. The white stripes near zero $n$ cover regions near the neutrality point, in which charge disorder becomes important and transport involves thermal broadening and other interaction effects ${ }^{12,13}$ beyond the scope of this work.
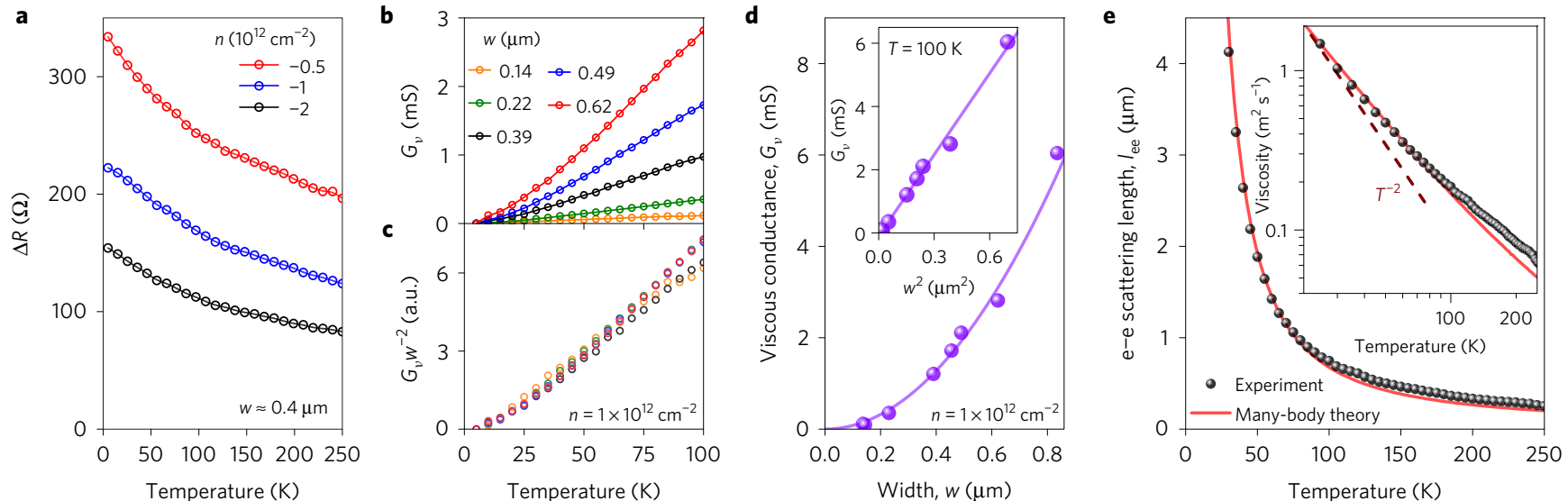

Figure 3 | Quantifying e-e interactions in graphene. a, $T$ dependence of the $P C$ resistance after subtracting the contribution from contact regions. b. Viscous conductance $G_{v}$ at a given $n$ for PCs with $w$ ranging between 0.1 and $0.6 \mu \mathrm{m}$. c, Data from $\mathbf{b}$ normalized by $w^{2}$. d, $G_{v}$ as a function of $w$ for given $T=100 \mathrm{~K}$ and $n=10^{12} \mathrm{~cm}^{-2}$. Solid curve: best fit to equation (2) yields $v \approx 0.16 \mathrm{~m}^{2} \mathrm{~s}^{-1}$, a value five orders of magnitude larger than the kinematic viscosity of water. Inset: same data as a function of $w^{2}$.e, $T$ dependence of the e-e scattering length found as $l_{\mathrm{ee}}=4 \mathrm{v} / \mathrm{v}_{\mathrm{F}}(\mathrm{symbols})$ for $n=10^{12} \mathrm{~cm}^{-2}$ and $w \approx 0.5 \mu \mathrm{m}$. Red curve: microscopic calculations of $l_{\mathrm{ee}}$ (Supplementary Section 6). Inset: $v(T)$ on a log-log scale. The data are from the main panel and colour-coded accordingly. The dashed line indicates the $1 / T^{2}$ dependence.

experimental data collapse onto a single curve (Fig. 3c). This scaling is starkly different from the Sharvin dependence $G_{\mathrm{b}} \propto w$ observed in the ballistic regime (Fig. 1d) and, more generally, from any known behaviour of electrical conductance that always varies linearly with the sample width. However, our result is in excellent agreement with equation (2) that suggests $G_{v} \propto w^{2}$. The $w^{2}$ scaling behaviour is further validated in Fig. 3d, lending strong support to our analysis.

The measured dependence $G_{v}(T)$ allows us to determine $v(T)$ and $l_{\mathrm{ee}}(T)$ using equation (2). The results are shown in Fig. $3 \mathrm{e}$ and compared with the calculations ${ }^{29}$ detailed in Supplementary Section 6. The agreement is surprisingly good (especially taking into account that neither experiment nor calculations use any fitting parameters) and holds for different PC devices and different carrier densities (Supplementary Section 7). We also note that the agreement is markedly better than the one achieved previously using measurements of $v$ in the vicinity geometry ${ }^{12}$, and even accommodates the fact that both experimental and theoretical curves in Fig. $3 e$ (inset) deviate from the $1 / T^{2}$ dependence expected for the normal Fermi liquid ${ }^{6,30}$. The deviations arise because temperatures $\sim 50-100 \mathrm{~K}$ are not insignificant with respect to the Fermi energy. Furthermore, our calculations in Fig. 3e stray slightly off the experimental curve above $100 \mathrm{~K}$. In fact, this is expected because, in the hydrodynamic regime $l_{\mathrm{ee}} \ll w$, the kinematic viscosity can no longer be expressed in terms of $l_{\mathrm{ee}}$ (as above) and 
requires a more accurate theoretical description using the two-body stress-stress response function ${ }^{29}$. Although the strong inequality $l_{\mathrm{ee}} \ll w$ is not reached in our experiments, the experimental data in Fig. 3e do tend in the expected direction (Supplementary Section 8).

To conclude, graphene constrictions provide a unique insight into the impact of e-e interactions on electron transport. The observed negative $T$ dependence of the point contact resistance, its superballistic values and the unusual $w^{2}$ scaling are clear indicators of the important role of e-e collisions in clean metals at elevated temperatures. Our analysis also offers a guide for unravelling intricate interaction effects contributing at the crossover between the ballistic and hydrodynamic transport regimes.

\section{Methods}

Methods, including statements of data availability and any associated accession codes and references, are available in the online version of this paper.

\section{Received 12 March 2017; accepted 14 July 2017;} published online 21 August 2017

\section{References}

1. Gurzhi, R. N. Minimum of resistance in impurity-fee conductors. Sov. Phys. JETP 17, 521-522 (1963).

2. Gurzhi, R. N. Hydrodynamic effects in solids at low temperature. Sov. Phys. Usp. 11, 255-270 (1968).

3. Govorov, A. O. \& Heremans, J. J. Hydrodynamic effects in interacting Fermi electron jets. Phys. Rev. Lett. 92, 026803 (2004).

4. Müller, M., Schmalian, J. \& Fritz, L. Graphene: a nearly perfect fluid. Phys. Rev. Lett. 103, 025301 (2009).

5. Mendoza, M., Herrmann, H. J. \& Succi, S. Preturbulent regimes in graphene flow. Phys. Rev. Lett. 106, 156601 (2011).

6. Forcella, D., Zaanen, J., Valentinis, D. \& van der Marel, D. Electromagnetic properties of viscous charged fluids. Phys. Rev. B 90, 035143 (2014).

7. Yu, Z. Z. et al. Negative temperature derivative of resistivity in thin potassium samples: the Gurzhi Effect? Phys. Rev. Lett. 52, 368-371 (1984).

8. de Jong, M. J. M. \& Molenkamp, L. W. Hydrodynamic electron flow in high-mobility wires. Phys. Rev. B 51, 13389-13402 (1995).

9. Renard, V. et al. Boundary-mediated electron-electron interactions in quantum point contacts. Phys. Rev. Lett. 100, 186801 (2008).

10. Nagaev, K. E. \& Kostyuchenko, T. V. Electron-electron scattering and magnetoresistance of ballistic microcontacts. Phys. Rev. B 81, 125316 (2010).

11. Melnikov, M. Y. et al. Influence of e-e scattering on the temperature dependence of the resistance of a classical ballistic point contact in a two-dimensional electron system. Phys. Rev. B 86, 075425 (2012).

12. Bandurin, D. A. et al. Negative local resistance caused by viscous electron backflow in graphene. Science 351, 1055-1058 (2016).

13. Crossno, J. et al. Observation of the Dirac fluid and the breakdown of the Wiedemann-Franz law in graphene. Science 351, 1058-1061 (2016).

14. Moll, P. J. W., Kushwaha, P., Nandi, N., Schmidt, B. \& Mackenzie, A. P. Evidence for hydrodynamic electron flow in $\mathrm{PdCoO}_{2}$. Science 351, 1061-1064 (2016).

15. Castro Neto, A. H., Guinea, F., Peres, N. M. R., Novoselov, K. S. \& Geim, A. K. The electronic properties of graphene. Rev. Mod. Phys. 81, 109-162 (2009).

16. Sharvin, Y. V. A possible method for studying Fermi surfaces. Sov. Phys. JETP 21, 655-656 (1965).

17. Beenakker, C. W. J. \& van Houten, H. Quantum transport in semiconductor nanostructures. Solid State Phys. 44, 1-228 (1991).
18. Guo, H., Ilseven, E., Falkovich, G. \& Levitov, L. Higher-than-ballistic conduction of viscous electron flows. Proc. Natl Acad. Sci. USA 114, 3068-3073 (2017).

19. Guo, H., Ilseven, E., Falkovich, G. \& Levitov, L. Stokes paradox, back reflections and interaction-enhanced conduction. Preprint at http://arxiv.org/abs/1612.09239 (2016).

20. Mayorov, A. S. et al. Micrometer-scale ballistic transport in encapsulated graphene at room temperature. Nano Lett. 11, 2396-2399 (2011).

21. Wang, L. et al. One-dimensional electrical contact to a two-dimensional material. Science 342, 614-617 (2013).

22. Torre, I., Tomadin, A., Geim, A. K. \& Polini, M. Nonlocal transport and the hydrodynamic shear viscosity in graphene. Phys. Rev. B 92, 165433 (2015).

23. Levitov, L. \& Falkovich, G. Electron viscosity, current vortices and negative nonlocal resistance in graphene. Nat. Phys. 12, 672-676 (2016).

24. Knudsen, M. Die Gesetze der Molekularströmung und der inneren Reibungsströmung der Gase durch Röhren (The laws of molecular flow and of inner frictional flow of gases through tubes). Ann. Phys. 28, 75-130 (1909).

25. Tombros, N. et al. Quantized conductance of a suspended graphene nanoconstriction. Nat. Phys. 7, 697-700 (2011).

26. Terrés, B. et al. Size quantization of Dirac fermions in graphene constrictions. Nat. Commun. 7, 11528 (2016).

27. Kotov, V. N., Uchoa, B., Pereira, V. M., Guinea, F. \& Castro Neto, A. H. Electron-electron interactions in graphene: Current status and perspectives. Rev. Mod. Phys. 84, 1067-1125 (2012).

28. de Jong, M. J. M. Transition from Sharvin to Drude resistance in high-mobility wires. Phys. Rev. B 49, 7778-7781 (1994).

29. Principi, A., Vignale, G., Carrega, M. \& Polini, M. Bulk and shear viscosities of the 2D electron liquid in a doped graphene sheet. Phys. Rev. B 93, 125410 (2016)

30. Polini, M. \& Vignale, G. The quasiparticle lifetime in a doped graphene sheet. Preprint at http://arxiv.org/abs/1404.5728 (2014).

\section{Acknowledgements}

This work was supported by Engineering and Physical Sciences Research Council, Graphene Flagship, the Royal Society and Lloyd's Register Foundation. L.S.L. acknowledges support from the Center for Integrated Quantum Materials under NSF award 1231319, the Center for Excitonics, an Energy Frontier Research Center funded by the US Department of Energy, Office of Science, Basic Energy Sciences, under award DESC0001088, and MIT-Israel Seed Fund. G.F. acknowledges ISF grant 882 and RSF grant 14-22-00259. A.P. was supported by ERC Advanced Grant FEMTO/NANO and Spinoza Prize. M.P. acknowledges Fondazione Istituto Italiano di Tecnologia and the European Union's Horizon 2020 programme under grant 696656 'GrapheneCorel'. D.A.B. and I.V.G. thank the Marie Curie programme SPINOGRAPH. G.H.A. was supported by EPSRC grant EP/M507969. R.K.K. acknowledges support from Doctoral Training Centre NOWNANO. The authors would like to thank E. Khestanova for the help with AFM measurements.

\section{Author contributions}

A.K.G., L.S.L. and M.P. designed and supervised the project. Y.C., G.H.A. and M.B.S. fabricated the studied devices. T.T. and K.W. provided quality boron-nitride crystals. Transport measurements and data analysis were carried out by R.K.K. and D.A.B. Theory analysis was done by F.M.D.P., A.P., H.G., G.F., L.S.L. and M.P. R.K.K., D.A.B., L.S.L., M.P. and A.K.G. wrote the manuscript. L.S.L. wrote Supplementary Section 4. A.P. and M.P. wrote Supplementary Sections 6 and 8. L.A.P. and I.V.G. provided experimental support and contributed to writing the manuscript. All authors contributed to discussions.

\section{Additional information}

Supplementary information is available in the online version of the paper. Reprints and permissions information is available online at www.nature.com/reprints. Publisher's note: Springer Nature remains neutral with regard to jurisdictional claims in published maps and institutional affiliations. Correspondence and requests for materials should be addressed to A.K.G.

\section{Competing financial interests}

The authors declare no competing financial interests. 


\section{Methods}

Our devices were made from encapsulated graphene heterostructures using electron-beam lithography and standard microfabrication procedures (Supplementary Section 1). The constriction widths were determined using Bruker's FastScan atomic force microscope. The electrical measurements were carried out in a variable temperature insert employing lock-in techniques at low frequencies $(10-30 \mathrm{~Hz})$ and with excitation currents of $0.1-1 \mu \mathrm{A}$.

Data availability. The data that support the plots within this paper and other findings of this study are available from the corresponding author upon reasonable request. 